\title{
Number of connected spanning subgraphs on the Sierpinski gasket
}

\author{
Shu-Chiuan Chang ${ }^{1}$ and Lung-Chi Chen ${ }^{2}$ \\ ${ }^{1}$ Department of Physics, National Cheng Kung University, Tainan 70101, Taiwan \\ ${ }^{2}$ Department of Mathematics, Fu Jen Catholic University, Taipei 24205, Taiwan
}

received June 4, 2008, revised January 13, 2009, accepted January 14, 2009.

\begin{abstract}
We study the number of connected spanning subgraphs $f_{d, b}(n)$ on the generalized Sierpinski gasket $S G_{d, b}(n)$ at stage $n$ with dimension $d$ equal to two, three and four for $b=2$, and layer $b$ equal to three and four for $d=2$. The upper and lower bounds for the asymptotic growth constant, defined as $z_{S G_{d, b}}=\lim _{v \rightarrow \infty} \ln f_{d, b}(n) / v$ where $v$ is the number of vertices, on $S G_{2, b}(n)$ with $b=2,3,4$ are derived in terms of the results at a certain stage. The numerical values of $z_{S G_{d, b}}$ are obtained.
\end{abstract}

Keywords: Connected spanning subgraphs, Sierpinski gasket, recursion relations, asymptotic growth constant

\section{Introduction}

The enumeration of the number of connected spanning subgraphs $N_{C S S G}(G)$ on a graph $G$ is a problem of interest in mathematics [Liu and Chow(1983)]. It is well known that the number of connected subgraphs is given by the Tutte polynomial $T(G, x, y)$ evaluated at $x=1, y=2$ [Welsh(1993)]. Alternatively, it corresponds to the partition function of the $q$-state Potts model in statistical mechanics with $q=0$ and the temperature variable $v=e^{\beta J}-1=1$, where $J$ is the spin-spin coupling and $\beta=\left(k_{B} T\right)^{-1}$. Some recent studies on the enumeration of connected spanning subgraphs and the calculation of their asymptotic growth constants on regular lattices were carried out in Refs. [Shrock(2000), Chang and Shrock(2000), Chang and Shrock(2001)(a), Chang and Shrock(2001)(b), Chang and Shrock(2001)(c)]. It is of interest to consider connected spanning subgraphs on self-similar fractal lattices which have scaling invariance rather than translational invariance. Fractals are geometric structures of (generally noninteger) Hausdorff dimension realized by repeated construction of an elementary shape on progressively smaller length scales [Mandelbrot(1982), Falconer(2003)]. A well-known example of a fractal is the Sierpinski gasket. We shall derive the recursion relations for the numbers of connected spanning subgraphs on the Sierpinski gasket with dimension equal to two, three and four, and determine the asymptotic growth constants. We shall also consider the number of connected spanning subgraphs on the generalized Sierpinski gasket with dimension equal to two.

1365-8050 @ 2009 Discrete Mathematics and Theoretical Computer Science (DMTCS), Nancy, France 


\section{Preliminaries}

We first recall some relevant definitions for connected spanning subgraphs and the Sierpinski gasket in this section. A connected graph (without loops) $G=(V, E)$ is defined by its vertex (site) and edge (bond) sets $V$ and $E$ [Biggs(1993), Harary(1969)]. Let $v(G)=|V|$ be the number of vertices and $e(G)=|E|$ the number of edges in $G$. A spanning subgraph $G^{\prime}$ is a subgraph of $G$ with the same vertex set $V$ and an edge set $E^{\prime} \subseteq E$. A connected spanning subgraph on $G$ is a spanning subgraph of $G$ that remains connected. In general, there can be cycles in a connected spanning subgraph. It is called a spanning tree when there is no cycles. The degree or coordination number $k_{i}$ of a vertex $v_{i} \in V$ is the number of edges attached to it. A $k$-regular graph is a graph with the property that each of its vertices has the same degree $k$. In general, one can associate an edge weight $x_{i j}$ to each edge connecting adjacent vertices $v_{i}$ and $v_{j}$. For simplicity, all edge weights are set to one throughout this paper.

Consider an infinite sequence of graphs $\{G(n)\}$ with $v(G(n)) \rightarrow \infty$ as $n \rightarrow \infty$. If the number of connected spanning subgraphs $N_{C S S G}(G(n))$ grows exponentially with $v(G(n))$ when $n \rightarrow \infty$ as

$$
N_{C S S G}(G(n))=\exp \left[z_{G} v(G(n))+o(v(G(n)))\right],
$$

where $\lim _{n \rightarrow \infty} o(v(G(n))) / v(G(n))=0$, then the constant $z_{G}$ can describe this exponential growth:

$$
z_{G}=\lim _{n \rightarrow \infty} \frac{\ln N_{C S S G}(G(n))}{v(G(n))}
$$

which is the entropy per site in statistical mechanics. Here $G$, when used as a subscript in this manner, implicitly refers to the thermodynamic limit.

The construction of the two-dimensional Sierpinski gasket $S G_{2}(n)$ at stage $n$ is shown in Fig. 1 At stage $n=0$, it is an equilateral triangle; while stage $(n+1)$ is obtained by the juxtaposition of three $n$-stage structures. In general, the Sierpinski gaskets $S G_{d}$ can be built in any Euclidean dimension $d$ with fractal dimension $D=\ln (d+1) / \ln 2$ [Gefen and Aharony(1981)] . For the Sierpinski gasket $S G_{d}(n)$, the numbers of edges and vertices are given by

$$
\begin{gathered}
e\left(S G_{d}(n)\right)=\left(\begin{array}{c}
d+1 \\
2
\end{array}\right)(d+1)^{n}=\frac{d}{2}(d+1)^{n+1}, \\
v\left(S G_{d}(n)\right)=\frac{d+1}{2}\left[(d+1)^{n}+1\right] .
\end{gathered}
$$

Except the $(d+1)$ outmost vertices which have degree $d$, all other vertices of $S G_{d}(n)$ have degree $2 d$. In the large $n$ limit, $S G_{d}$ is $2 d$-regular.

The Sierpinski gasket can be generalized, denoted by $S G_{d, b}(n)$, by introducing the side length $b$ which is an integer larger or equal to two [Hilfer and Blumen(1984)]. The generalized Sierpinski gasket at stage $(n+1)$ is constructed from $b$ layers of stage $n$ hypertetrahedrons. The two-dimensional $S G_{2, b}(n)$ with $b=3$ at stage $n=1,2$ and $b=4$ at stage $n=1$ are illustrated in Fig. 2. The ordinary Sierpinski gasket $S G_{d}(n)$ corresponds to the $b=2$ case, where the index $b$ is neglected for simplicity. The Hausdorff dimension for $S G_{d, b}$ is given by $D=\ln \left(\begin{array}{c}b+d-1 \\ d\end{array}\right) / \ln b$ [Hilfer and Blumen(1984)]. Notice that $S G_{d, b}$ is not $k$-regular even in the thermodynamic limit. 


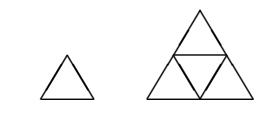

$S G_{2}(0) \quad S G_{2}(1)$

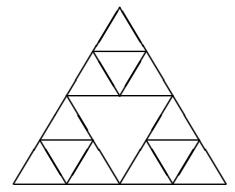

$S G_{2}(2)$

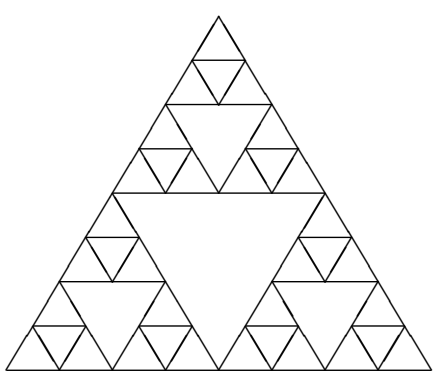

$S G_{2}(3)$

Fig. 1: The first four stages $n=0,1,2,3$ of the two-dimensional Sierpinski gasket $S G_{2}(n)$.

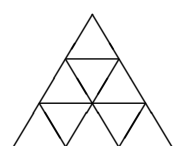

$S G_{2,3}(1)$

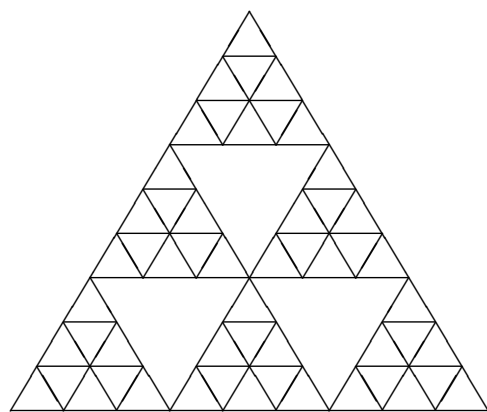

$S G_{2,3}(2)$

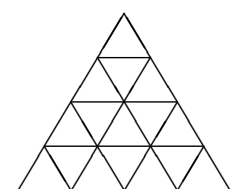

$S G_{2,4}(1)$

Fig. 2: The generalized two-dimensional Sierpinski gasket $S G_{2, b}(n)$ with $b=3$ at stage $n=1,2$ and $b=4$ at stage $n=1$. 


\section{The number of connected spanning subgraphs on $S G_{2}(n)$}

In this section we derive the asymptotic growth constant for the number of connected spanning subgraphs on the two-dimensional Sierpinski gasket $S G_{2}(n)$ in detail. Let us start with the definitions of the quantities to be used.

Definition 3.1 Consider the generalized two-dimensional Sierpinski gasket $S G_{2, b}(n)$ at stage $n$.

(i) Define $f_{2, b}(n) \equiv N_{C S S G}\left(S G_{2, b}(n)\right)$ as the number of connected spanning subgraphs.

(ii) Define $g_{2, b}(n)$ as the number of spanning subgraphs with two connected components such that one certain outmost vertex, say the topmost vertex as illustrated in Fig. 3 for ordinary Sierpinski gasket, belongs to one component and the other two outmost vertices belong to another component.

(iii) Define $h_{2, b}(n)$ as the number of spanning subgraphs with three connected components such that each of the outmost vertices belongs to a different component.

Since we only consider the ordinary Sierpinski gasket in this section, we use the notations $f_{2}(n)$, $g_{2}(n)$ and $h_{2}(n)$ for simplicity. They are illustrated in Fig. 3, where only the outmost vertices are shown. Because of rotational symmetry, there are three possible $g_{2}(n)$. The initial values at stage zero are $f_{2}(0)=$ $4, g_{2}(0)=1$ and $h_{2}(0)=1$. The purpose of this section is to obtain the asymptotic behavior of $f_{2}(n)$ as follows. The three quantities $f_{2}(n), g_{2}(n)$ and $h_{2}(n)$ satisfy recursion relations.
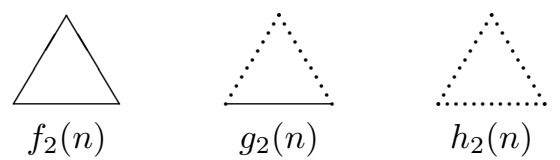

Fig. 3: Illustration for the connected spanning subgraphs $f_{2}(n), g_{2}(n)$ and $h_{2}(n)$. The two outmost vertices at the ends of a solid line belong to one component, while the two outmost vertices at the ends of a dot line belong to separated components.

Lemma 3.1 For any non-negative integer $n$,

$$
\begin{gathered}
f_{2}(n+1)=f_{2}^{3}(n)+6 f_{2}^{2}(n) g_{2}(n), \\
g_{2}(n+1)=f_{2}^{2}(n) g_{2}(n)+f_{2}^{2}(n) h_{2}(n)+7 f_{2}(n) g_{2}^{2}(n), \\
h_{2}(n+1)=3 f_{2}(n) g_{2}^{2}(n)+12 f_{2}(n) g_{2}(n) h_{2}(n)+14 g_{2}^{3}(n) .
\end{gathered}
$$




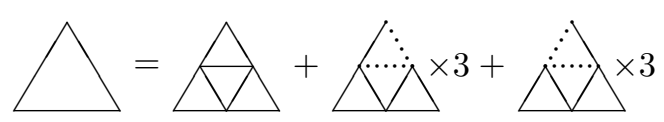

Fig. 4: Illustration for the expression of $f_{2}(n+1)$. The multiplication of three on the right-hand-side corresponds to the three possible orientations of $S G_{2}(n+1)$.

Proof: The Sierpinski gasket $S G_{2}(n+1)$ is composed of three $S G_{2}(n)$ with three pairs of vertices identified. The number $f_{2}(n+1)$ consists of one configuration where all three $S G_{2}(n)$ belong to the class that is enumerated by $f_{2}(n)$, and six configurations where one of the $S G_{2}(n)$ belongs to the class enumerated by $g_{2}(n)$ and the other two belong to the class enumerated by $f_{2}(n)$ as illustrated in Fig. 4 . Eq. (5) is verified by adding these configurations.

Similarly, $g_{2}(n+1)$ and $h_{2}(n+1)$ for $S G_{2}(n+1)$ can be obtained with appropriate configurations of its three constituting $S G_{2}(n)$ as illustrated in Figs. 5 and 6 to verify Eqs. (6) and (7), respectively.

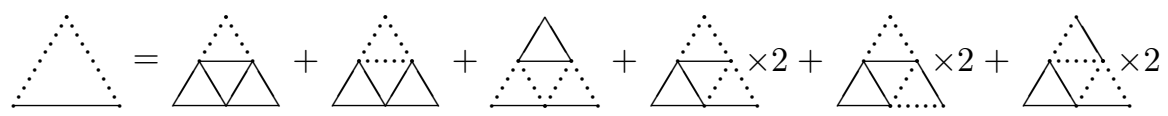

Fig. 5: Illustration for the expression of $g_{2}(n+1)$. The multiplication of two on the right-hand-side corresponds to the reflection symmetry with respect to the central vertical axis.

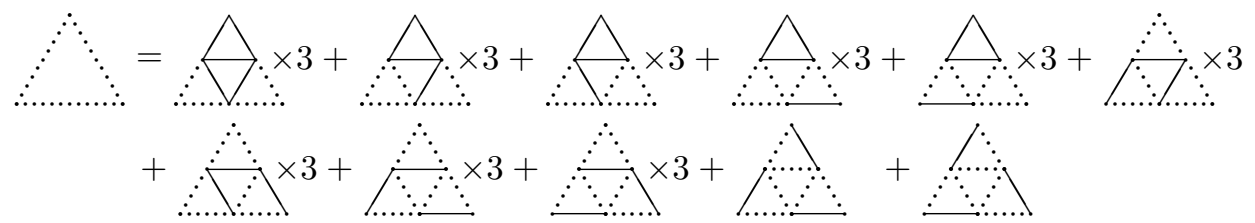

Fig. 6: Illustration for the expression of $h_{2}(n+1)$. The multiplication of three on the right-hand-side corresponds to the three possible orientations $S G_{2}(n+1)$.

The values of $f_{2}(n), g_{2}(n), h_{2}(n)$ for small $n$ can be evaluated recursively by Eqs. (5), (6), (7) as listed in Table 1 These numbers grow exponentially, and do not have simple integer factorizations, in contrast to the corresponding results for the number of spanning trees [Chang et al.(2007)]. To estimate the value of the asymptotic growth constant defined in Eq. (2), we need the following lemmas. For the generalized two-dimensional Sierpinski gasket $S G_{2, b}(n)$, define the ratios

$$
\alpha_{2, b}(n)=\frac{f_{2, b}(n)}{g_{2, b}(n)}, \quad \beta_{2, b}(n)=\frac{g_{2, b}(n)}{h_{2, b}(n)},
$$


where $n$ is a non-negative integer. For the ordinary Sierpinski gasket in this section, they are simplified to be $\alpha_{2}(n), \beta_{2}(n)$ and their values for small $n$ are listed in Table 2

Tab. 1: The first few values of $f_{2}(n), g_{2}(n), h_{2}(n)$.

\begin{tabular}{|c||r|r|r|r|}
\hline \hline$n$ & 0 & 1 & 2 & 3 \\
\hline \hline$f_{2}(n)$ & 4 & 160 & $13,312,000$ & $10,293,452,839,321,600,000,000$ \\
\hline$g_{2}(n)$ & 1 & 60 & $7,462,400$ & $8,864,355,990,896,640,000,000$ \\
\hline$h_{2}(n)$ & 1 & 74 & $13,276,800$ & $23,868,720,258,482,176,000,000$ \\
\hline \hline
\end{tabular}

Tab. 2: The first few values of $\alpha_{2}(n), \beta_{2}(n)$. The last digits given are rounded off.

\begin{tabular}{|c||r|r|r|r|r|}
\hline \hline$n$ & 0 & 1 & 2 & 3 & 4 \\
\hline \hline$\alpha_{2}(n)$ & 4 & 2.66666666666667 & 1.78387650085763 & 1.16121835019855 & 0.736689163182441 \\
\hline$\beta_{2}(n)$ & 1 & 0.810810810810811 & 0.562063147746445 & 0.371379608747416 & 0.238302798822389 \\
\hline \hline
\end{tabular}

Lemma 3.2 For any $n \geq 0$,

$$
3 \beta_{2}(n) \leq \alpha_{2}(n) \leq 4 \beta_{2}(n) .
$$

The ratios $\alpha_{2}(n)$ and $\beta_{2}(n)$ are both strictly decreasing sequences with the limits

$$
\lim _{n \rightarrow \infty} \alpha_{2}(n)=\lim _{n \rightarrow \infty} \beta_{2}(n)=0 .
$$

Proof: It is clear that $\alpha_{2}(n)$ and $\beta_{2}(n)$ cannot be negative. By Eqs. (5) - (7), we have

$$
\begin{gathered}
\frac{f_{2}(n+1)}{f_{2}^{2}(n) g_{2}(n)}=6+\alpha_{2}(n), \\
\frac{g_{2}(n+1)}{f_{2}(n) g_{2}^{2}(n)}=\frac{\alpha_{2}(n)}{\beta_{2}(n)}+7+\alpha_{2}(n), \\
\frac{h_{2}(n+1)}{g_{2}^{3}(n)}=12 \frac{\alpha_{2}(n)}{\beta_{2}(n)}+14+3 \alpha_{2}(n) .
\end{gathered}
$$

Therefore,

$$
\alpha_{2}(n+1)=\frac{\alpha_{2}(n) \beta_{2}(n)\left[6+\alpha_{2}(n)\right]}{\alpha_{2}(n)+7 \beta_{2}(n)+\alpha_{2}(n) \beta_{2}(n)}=\alpha_{2}(n)-\frac{\alpha_{2}(n)\left[\alpha_{2}(n)+\beta_{2}(n)\right]}{\alpha_{2}(n)+7 \beta_{2}(n)+\alpha_{2}(n) \beta_{2}(n)},
$$


which shows that $\alpha_{2}(n)$ is strictly decreasing. Similarly, we have

$\beta_{2}(n+1)=\frac{\alpha_{2}(n)\left[\alpha_{2}(n)+7 \beta_{2}(n)+\alpha_{2}(n) \beta_{2}(n)\right]}{12 \alpha_{2}(n)+14 \beta_{2}(n)+3 \alpha_{2}(n) \beta_{2}(n)}=\frac{\alpha_{2}(n)}{3}-\frac{\alpha_{2}(n)\left[3 \alpha_{2}(n)-7 \beta_{2}(n) / 3\right]}{12 \alpha_{2}(n)+14 \beta_{2}(n)+3 \alpha_{2}(n) \beta_{2}(n)}$.

With the initial values given in Table $2,3 \beta_{2}(n) \leq \alpha_{2}(n)$ is proved by induction. By Eqs. 14 and (15),

$$
\begin{aligned}
& 4 \beta_{2}(n+1)-\alpha_{2}(n+1) \\
= & \frac{\alpha_{2}(n)}{3}+\frac{\alpha_{2}(n)\left[\alpha_{2}(n)+\beta_{2}(n)\right]}{\alpha_{2}(n)+7 \beta_{2}(n)+\alpha_{2}(n) \beta_{2}(n)}-\frac{\alpha_{2}(n)\left[12 \alpha_{2}(n)-28 \beta_{2}(n) / 3\right]}{12 \alpha_{2}(n)+14 \beta_{2}(n)+3 \alpha_{2}(n) \beta_{2}(n)} \\
= & \frac{\alpha_{2}(n) X(n)}{3\left[\alpha_{2}(n)+7 \beta_{2}(n)+\alpha_{2}(n) \beta_{2}(n)\right]\left[12 \alpha_{2}(n)+14 \beta_{2}(n)+3 \alpha_{2}(n) \beta_{2}(n)\right]},
\end{aligned}
$$

where

$$
X(n)=12 \alpha_{2}^{2}(n)+48 \beta_{2}(n)\left[7 \beta_{2}(n)-\alpha_{2}(n)\right]+12 \alpha_{2}(n) \beta_{2}(n)\left[6 \beta_{2}(n)-\alpha_{2}(n)\right]+3 \alpha_{2}^{2}(n) \beta_{2}^{2}(n) \geq 0
$$

such that $\alpha_{2}(n) \leq 4 \beta_{2}(n)$ is proved again by induction. Eq. 15 ) can be rewritten as

$\beta_{2}(n+1)=\beta_{2}(n)-\frac{\alpha_{2}(n)\left[4 \beta_{2}(n)-\alpha_{2}(n)\right]\left[1+3 \beta_{2}(n) / 4\right]+\alpha_{2}(n) \beta_{2}(n)\left[1-\alpha_{2}(n) / 4\right]+14 \beta_{2}^{2}(n)}{12 \alpha_{2}(n)+14 \beta_{2}(n)+3 \alpha_{2}(n) \beta_{2}(n)}$,

which shows that $\beta_{2}(n)$ is strictly decreasing since $\alpha_{2}(n)$ is less than four, $i$.e. its initial value, for all $n \geq 1$. Eq. (14) can be rewritten as

$$
\alpha_{2}(n+1)=\frac{6 \alpha_{2}(n)}{7}\left[1-\frac{\alpha_{2}(n)\left[1-\beta_{2}(n) / 6\right]}{\alpha_{2}(n)+7 \beta_{2}(n)+\alpha_{2}(n) \beta_{2}(n)}\right]
$$

which is always less than $6 \alpha_{2}(n) / 7$ since $\beta_{2}(n)$ is less than one, $i$.e. its initial value, for all $n \geq 1$ such that $\lim _{n \rightarrow \infty} \alpha_{2}(n)$ is zero. Finally, since $\beta_{2}(n) \leq \alpha_{2}(n) / 3, \lim _{n \rightarrow \infty} \beta_{2}(n)$ is zero, and the proof is completed.

We notice that the convergences of $\alpha_{2}(n)$ and $\beta_{2}(n)$ to zero as $n$ increases are not rapid. The inequality $3 \beta_{2}(n) \leq \alpha_{2}(n)$ can be improved a bit, and we state it as the following lemma.

Lemma 3.3 For any $n \geq n_{0}$,

$$
3 \beta_{2}(n)+\frac{\alpha_{2}^{2}(n)}{27} \leq \alpha_{2}(n),
$$

where $n_{0}=\inf \left\{n: \alpha_{2}(n) \leq 3 / 4, \beta_{2}(n) \leq 1 / 4\right\}=4$.

Proof: By Eqs. (14) and 15, we have

$$
\alpha_{2}(n+1)-3 \beta_{2}(n+1)-\frac{\alpha_{2}^{2}(n+1)}{27}
$$




$$
\begin{aligned}
= & \frac{\alpha_{2}(n)\left[9 \alpha_{2}(n)-7 \beta_{2}(n)\right]}{12 \alpha_{2}(n)+14 \beta_{2}(n)+3 \alpha_{2}(n) \beta_{2}(n)}-\frac{\alpha_{2}(n)\left[\alpha_{2}(n)+\beta_{2}(n)\right]}{\alpha_{2}(n)+7 \beta_{2}(n)+\alpha_{2}(n) \beta_{2}(n)} \\
& -\frac{\alpha_{2}^{2}(n) \beta_{2}^{2}(n)\left[6+\alpha_{2}(n)\right]^{2}}{27\left[\alpha_{2}(n)+7 \beta_{2}(n)+\alpha_{2}(n) \beta_{2}(n)\right]^{2}} \\
= & \frac{\alpha_{2}(n) Y(n)}{\left[\alpha_{2}(n)+7 \beta_{2}(n)+\alpha_{2}(n) \beta_{2}(n)\right]^{2}\left[12 \alpha_{2}(n)+14 \beta_{2}(n)+3 \alpha_{2}(n) \beta_{2}(n)\right]},
\end{aligned}
$$

where

$$
\begin{aligned}
Y(n)= & {\left[\alpha_{2}(n)+7 \beta_{2}(n)+\alpha_{2}(n) \beta_{2}(n)\right]\left\{3\left[\alpha_{2}(n)-3 \beta_{2}(n)\right]\left[4 \beta_{2}(n)-\alpha_{2}(n)\right]\right.} \\
& \left.+3 \beta_{2}(n)\left[\alpha_{2}(n)-3 \beta_{2}(n)-\frac{\alpha_{2}^{2}(n)}{27}\right]\left[3+2 \alpha_{2}(n)\right]\right\} \\
& +\frac{\alpha_{2}(n) \beta_{2}(n)}{27}\left[9 \alpha_{2}^{2}(n)+1008 \beta_{2}^{2}(n)-153 \alpha_{2}(n) \beta_{2}(n)+6 \alpha_{2}^{3}(n)-93 \alpha_{2}^{2}(n) \beta_{2}(n)\right. \\
& \left.-60 \alpha_{2}(n) \beta_{2}^{2}(n)-6 \alpha_{2}^{3}(n) \beta_{2}(n)-50 \alpha_{2}^{2}(n) \beta_{2}^{2}(n)-3 \alpha_{2}^{3}(n) \beta_{2}^{2}(n)\right] \\
\geq & \frac{\alpha_{2}(n) \beta_{2}(n)}{27}\left\{3 \alpha_{2}(n)\left[\alpha_{2}(n)-3 \beta_{2}(n)\right]\left[3+2 \alpha_{2}(n)\right]+252 \beta_{2}(n)\left[4 \beta_{2}(n)-\alpha_{2}(n)\right]\right. \\
& \left.+\alpha_{2}(n) \beta_{2}(n)\left[126-75 \alpha_{2}(n)-6 \alpha_{2}^{2}(n)-60 \beta_{2}(n)-50 \alpha_{2}(n) \beta_{2}(n)-3 \alpha_{2}^{2}(n) \beta_{2}(n)\right]\right\} .
\end{aligned}
$$

Because $Y(n)$ is positive whenever $\alpha_{2}(n) \leq 3 / 4$ and $\beta_{2}(n) \leq 1 / 4$, which is true for all $n \geq n_{0}=4$ by the previous lemma and Table 2 , the inequality is established.

We notice that although Eq. 20] is by no means optimum, it is enough for the following lemma.

Lemma 3.4 The sequence of the ratio $\left\{\alpha_{2}(n) / \beta_{2}(n)\right\}_{n=0}^{\infty}$ decreases monotonically with the limit

$$
\lim _{n \rightarrow \infty} \alpha_{2}(n) / \beta_{2}(n)=3 \text {. }
$$

Proof: The initial value of the ratio is $\alpha_{2}(0) / \beta_{2}(0)=4$. It is clear from Eq. 15 that in the large $n$ limit, the ratio $\alpha_{2}(n) / \beta_{2}(n)$ is equal to three. By Eqs. (14) and [15, we have

$$
\frac{\alpha_{2}(n)}{\beta_{2}(n)}-\frac{\alpha_{2}(n+1)}{\beta_{2}(n+1)}=\frac{Z(n)}{\beta_{2}(n)\left[\alpha_{2}(n)+7 \beta_{2}(n)+\alpha_{2}(n) \beta_{2}(n)\right]^{2}},
$$

where

$$
\begin{aligned}
Z(n)= & \alpha_{2}(n)\left[\alpha_{2}(n)+7 \beta_{2}(n)+\alpha_{2}(n) \beta_{2}(n)\right]^{2} \\
& -\beta_{2}^{2}(n)\left[6+\alpha_{2}(n)\right]\left[12 \alpha_{2}(n)+14 \beta_{2}(n)+3 \alpha_{2}(n) \beta_{2}(n)\right]
\end{aligned}
$$




$$
\begin{gathered}
=\left[\alpha_{2}(n)-3 \beta_{2}(n)\right]\left[2 \alpha_{2}^{2}(n) \beta_{2}(n)+\frac{32}{3} \alpha_{2}(n) \beta_{2}^{2}(n)+\alpha_{2}^{2}(n) \beta_{2}^{2}(n)\right] \\
+\left[\alpha_{2}(n)-3 \beta_{2}(n)-\frac{\alpha_{2}^{2}(n)}{27}\right]\left[\alpha_{2}^{2}(n)+17 \alpha_{2}(n) \beta_{2}(n)+28 \beta_{2}^{2}(n)\right] \\
+\frac{\alpha_{2}^{2}(n)}{27}\left[\alpha_{2}^{2}(n)+17 \alpha_{2}(n) \beta_{2}(n)+28 \beta_{2}^{2}(n)\right]-\frac{8}{3} \alpha_{2}^{2}(n) \beta_{2}^{2}(n) .
\end{gathered}
$$

With $\alpha_{2}(n) \geq 3 \beta_{2}(n), Z(n)$ is positive such that the sequence of the ratio decreases monotonically.

The general expressions for $f_{2}(n)$ and $g_{2}(n)$ can be written as follows.

Lemma 3.5 For a non-negative integer $m$ and any positive integer $n>m$,

$$
\begin{aligned}
f_{2}(n)= & f_{2}(m)^{\frac{3^{n-m}+1}{2}} g_{2}(m)^{\frac{3^{n-m}-1}{2}} \prod_{i=1}^{n-m}\left[6+\alpha_{2}(n-i)\right]^{\frac{3^{i-1}+1}{2}} \\
& \times \prod_{j=2}^{n-m}\left[7+\alpha_{2}(n-j)+\frac{\alpha_{2}(n-j)}{\beta_{2}(n-j)}\right]^{\frac{3^{j-1}-1}{2}}, \\
g_{2}(n)= & f_{2}(m)^{\frac{3^{n-m}-1}{2}} g_{2}(m)^{\frac{3^{n-m}+1}{2}} \prod_{i=2}^{n-m}\left[6+\alpha_{2}(n-i)\right]^{\frac{3^{i-1}-1}{2}} \\
& \times \prod_{j=1}^{n-m}\left[7+\alpha_{2}(n-j)+\frac{\alpha_{2}(n-j)}{\beta_{2}(n-j)}\right]^{\frac{3^{j-1}+1}{2}} .
\end{aligned}
$$

Here when $n-m=1$, the products with lower limit two are defined to be one.

Proof: It is clear from Eqs. (11) and $\sqrt{12}$ that $f_{2}(m+1)=f_{2}^{2}(m) g_{2}(m)\left[6+\alpha_{2}(m)\right]$ and $g_{2}(m+1)=$ $f_{2}(m) g_{2}^{2}(m)\left[7+\alpha_{2}(m)+\alpha_{2}(m) / \beta_{2}(m)\right]$. Consider Eqs. 26) and 27) hold for a certain positive integer $n=k$, then

$$
\begin{aligned}
f_{2}(k+1)= & f_{2}^{2}(k) g_{2}(k)\left[6+\alpha_{2}(k)\right] \\
= & f_{2}(m)^{3^{k-m}+1} g_{2}(m)^{3^{k-m}-1} \prod_{i=1}^{k-m}\left[6+\alpha_{2}(k-i)\right]^{3^{i-1}+1} \\
& \times \prod_{j=2}^{k-m}\left[7+\alpha_{2}(k-j)+\frac{\alpha_{2}(k-j)}{\beta_{2}(k-j)}\right]^{3^{j-1}-1} \\
& \times f_{2}(m)^{\frac{3^{k-m}-1}{2}} g_{2}(m)^{\frac{3^{k-m}+1}{2}} \prod_{i=2}^{k-m}\left[6+\alpha_{2}(k-i)\right]^{\frac{3^{i-1}-1}{2}}
\end{aligned}
$$




$$
\begin{aligned}
& \times \prod_{j=1}^{k-m}\left[7+\alpha_{2}(k-j)+\frac{\alpha_{2}(k-j)}{\beta_{2}(k-j)}\right]^{\frac{3^{j-1}+1}{2}}\left[6+\alpha_{2}(k)\right] \\
= & f_{2}(m)^{\frac{3^{k-m+1}+1}{2}} g_{2}(m)^{\frac{3^{k-m+1}-1}{2}}\left[6+\alpha_{2}(k)\right]\left[6+\alpha_{2}(k-1)\right]^{2} \\
& \times \prod_{i=2}^{k-m}\left[6+\alpha_{2}(k-i)\right]^{\frac{3^{i}+1}{2}}\left[7+\alpha_{2}(k-1)+\frac{\alpha_{2}(k-1)}{\beta_{2}(k-1)}\right] \\
& \times \prod_{j=2}^{k-m}\left[7+\alpha_{2}(k-j)+\frac{\alpha_{2}(k-j)}{\beta_{2}(k-j)}\right]^{\frac{3^{j}-1}{2}} \\
= & f_{2}(m)^{\frac{3^{k-m+1}+1}{2}} g_{2}(m)^{\frac{3^{k-m+1}-1}{2}} \prod_{i=1}^{k-m+1}\left[6+\alpha_{2}(k+1-i)\right]^{\frac{3^{i-1}+1}{2}} \\
& k-m+1 \\
& \prod_{j=2}^{k-m+1}\left[7+\alpha_{2}(k+1-j)+\frac{\alpha_{2}(k+1-j)}{\beta_{2}(k+1-j)}\right]^{\frac{3^{j-1}-1}{2}},
\end{aligned}
$$

and Eq. 26] is proved by induction. Eq. 27] can be established by the same procedure.

From the above lemmas, we have the following bounds for the asymptotic growth constant.

Lemma 3.6 The asymptotic growth constant for the number of connected spanning subgraphs on $S G_{2}(n)$ is bounded:

$$
\frac{\ln \left[f_{2}(m) g_{2}(m)\right]+\frac{1}{2} \ln 60}{3^{m+1}} \leq z_{S G_{2}} \leq \frac{\ln \left[f_{2}(m) g_{2}(m)\right]+\frac{1}{2} \ln \left[6+\alpha_{2}(m)\right]\left[7+\alpha_{2}(m)+\frac{\alpha_{2}(m)}{\beta_{2}(m)}\right]}{3^{m+1}}
$$

where $m$ is a positive integer.

Proof: By Lemma 3.5, we have

$$
\ln f_{2}(n)=\frac{3^{n-m}+1}{2} \ln f_{2}(m)+\frac{3^{n-m}-1}{2} \ln g_{2}(m)+\Delta(n, m)
$$

where

$$
\Delta(n, m)=\sum_{i=1}^{n-m} \frac{3^{i-1}+1}{2} \ln \left[6+\alpha_{2}(n-i)\right]+\sum_{j=2}^{n-m} \frac{3^{j-1}-1}{2} \ln \left[7+\alpha_{2}(n-j)+\frac{\alpha_{2}(n-j)}{\beta_{2}(n-j)}\right] .
$$

We have shown that as $m$ increases, $\alpha_{2}(m)$ decreases to zero in Lemma 3.2 and $\alpha_{2}(m) / \beta_{2}(m)$ decreases to three in Lemma 3.4 such that

$$
\Delta(n, m) \leq \sum_{i=1}^{n-m} \frac{3^{i-1}+1}{2} \ln \left[6+\alpha_{2}(m)\right]+\sum_{j=2}^{n-m} \frac{3^{j-1}-1}{2} \ln \left[7+\alpha_{2}(m)+\frac{\alpha_{2}(m)}{\beta_{2}(m)}\right]
$$




$$
\begin{aligned}
= & \frac{1}{2}\left(\frac{3^{n-m}-1}{2}+n-m\right) \ln \left[6+\alpha_{2}(m)\right] \\
& +\frac{1}{2}\left(\frac{3^{n-m}-3}{2}-n+m+1\right) \ln \left[7+\alpha_{2}(m)+\frac{\alpha_{2}(m)}{\beta_{2}(m)}\right]
\end{aligned}
$$

and

$$
\begin{aligned}
\Delta(n, m) & \geq \sum_{i=1}^{n-m} \frac{3^{i-1}+1}{2} \ln 6+\sum_{j=2}^{n-m} \frac{3^{j-1}-1}{2} \ln 10 \\
& =\frac{1}{2}\left(\frac{3^{n-m}-1}{2}+n-m\right) \ln 6+\frac{1}{2}\left(\frac{3^{n-m}-3}{2}-n+m+1\right) \ln 10 .
\end{aligned}
$$

With the definition for $z_{S G_{2}}$ given in Eq. 22 and the number of vertices of $S G_{2}(n)$ is $3\left(3^{n}+1\right) / 2$ by Eq. (4), the proof is completed.

As $m$ increases, the difference between the upper and lower bounds in Eq. 29] is less than $3^{-m}$ and converges to zero. We calculate the number of connected spanning subgraphs $f_{2}(m)$ up to $m=15$, and we have the following proposition.

Proposition 3.1 The asymptotic growth constant for the number of connected spanning subgraphs on the two-dimensional Sierpinski gasket $S G_{2}(n)$ in the large $n$ limit exists with value $z_{S G_{2}}=1.276495930 \ldots$.

Without going into details, we state here without proof that the bounds can be improved. For a nonnegative integer $m$ and any positive integer $n>m$, the tighter bounds for $\alpha_{2}(n)$ are

$$
d^{n-m}(m) \leq \alpha_{2}(n) \leq c^{n-m}(m)
$$

where

$$
c(m)=\frac{6+\alpha_{2}(m)}{10+\alpha_{2}(m)}, \quad d(m)=\frac{6 \beta_{2}(m)}{\alpha_{2}(m)+7 \beta_{2}(m)}
$$

It can be shown that

$$
z_{S G_{2}} \leq \frac{1}{3^{m+1}}\left\{\ln \left[f_{2}(m) g_{2}(m)\right]+\frac{1}{2} \ln \left[42+6 \frac{\alpha_{2}(m)}{\beta_{2}(m)}\right]+\frac{\alpha_{2}(m)}{[3-c(m)]}\left[\frac{1}{6}+\frac{\beta_{2}(m)}{7 \beta_{2}(m)+\alpha_{2}(m)}\right]\right\}
$$

and

$$
z_{S G_{2}} \geq \frac{1}{3^{m+1}}\left\{\ln \left[f_{2}(m) g_{2}(m)\right]+\frac{\ln 60}{2}+\frac{4 \alpha_{2}(m)}{15[3-d(m)]}-\frac{17 \alpha_{2}^{2}(m)}{900\left[3-d^{2}(m)\right]}\right\},
$$

so that the asymptotic growth constant is $z_{S G_{2}}=1.27649593067 \ldots$. We notice that the numerical value can also be estimated by the standard technique given in [Aho and Sloane(1973)] with similar rate of convergence. 
The asymptotic behaviors of $f_{2}(n), g_{2}(n)$ and $h_{2}(n)$ can be derived as follows (Aho and Sloane(1973), Teufl and Wagner(2007)]. Rewriting Eqs. (14), 15p into

$$
\begin{aligned}
\frac{\alpha_{2}(n+1)}{\alpha_{2}(n)} & =\frac{6+\alpha_{2}(n)}{\frac{\alpha_{2}(n)}{\beta_{2}(n)}+7+\alpha_{2}(n)}, \\
\frac{\beta_{2}(n+1)}{\beta_{2}(n)} & =\frac{\frac{\alpha_{2}(n)}{\beta_{2}(n)}+7+\alpha_{2}(n)}{12+14 \frac{\alpha_{2}(n)}{\beta_{2}(n)}+3 \beta_{2}(n)}
\end{aligned}
$$

and using Lemmas 3.2 and 3.4 , it is not hard to see that both $(5 / 3)^{n} \alpha_{2}(n)$ and $(5 / 3)^{n} \beta_{2}(n)$ tend to (positive, finite) limits. By employing Eq. (6) repeatedly, we have

$$
\ln g_{2}(n)=3^{n-m} \ln g_{2}(m)+\sum_{j=m+1}^{n} 3^{n-j} \ln \left\{\alpha_{2}(j)\left[\alpha_{2}(j)+\frac{\alpha_{2}(j)}{\beta_{2}(j)}+7\right]\right\}
$$

for a certain $m$ less than $n$. When both $n$ and $m$ are large, $\ln g_{2}(n)$ is given approximately by

$$
\ln g_{2}(n) \sim 3^{n-m} \ln g_{2}(m)+\sum_{j=m+1}^{n} 3^{n-j}[a(j)+j \ln (3 / 5)],
$$

where $a(j)$ is close to a constant for all the $j$ values. Carrying out the summation

$$
\sum_{j=m+1}^{n} 3^{n-j} j=3^{n-m}\left(\frac{m}{2}+\frac{3}{4}\right)-\frac{3}{4}-\frac{n}{2},
$$

it follows that

$$
g_{2}(n) \sim C\left(g_{2}\right)\left(\frac{5}{3}\right)^{n / 2} A_{2}^{3^{n}},
$$

where $C\left(g_{2}\right)$ and $A_{2}$ are constants with $z_{S G_{2}}=(2 / 3) \ln A_{2}$. Similarly, we have

$$
f_{2}(n) \sim C\left(f_{2}\right)\left(\frac{3}{5}\right)^{n / 2} A_{2}^{3^{n}}, \quad h_{2}(n) \sim C\left(h_{2}\right)\left(\frac{5}{3}\right)^{3 n / 2} A_{2}^{3^{n}},
$$

where $C\left(f_{2}\right), C\left(h_{2}\right)$ are constants.

\section{The number of connected spanning subgraphs on $S G_{2, b}(n)$ with $b=3,4$}

The method given in the previous section can be applied to the number of connected spanning subgraphs on $S G_{d, b}(n)$ with larger values of $d$ and $b$. The number of configurations to be considered increases as $d$ and $b$ increase, and the recursion relations must be derived individually for each $d$ and $b$. In this section, we consider the generalized two-dimensional Sierpinski gasket $S G_{2, b}(n)$ with the number of layers $b$ equal to three and four. For $S G_{2,3}(n)$, the numbers of edges and vertices are given by

$$
e\left(S G_{2,3}(n)\right)=3 \times 6^{n},
$$




$$
v\left(S G_{2,3}(n)\right)=\frac{7 \times 6^{n}+8}{5},
$$

where the three outmost vertices have degree two. There are $\left(6^{n}-1\right) / 5$ vertices of $S G_{2,3}(n)$ with degree six and $6\left(6^{n}-1\right) / 5$ vertices with degree four. The initial values for the number of connected spanning subgraphs are the same as for $S G_{2}: f_{2,3}(0)=4, g_{2,3}(0)=1$ and $h_{2,3}(0)=1$. By the method illustrated in the previous section, we obtain the following recursion relations for any non-negative integer $n$.

$$
\begin{gathered}
f_{2,3}(n+1)=f_{2,3}^{6}(n)+15 f_{2,3}^{5}(n) g_{2,3}(n)+3 f_{2,3}^{5}(n) h_{2,3}(n)+78 f_{2,3}^{4}(n) g_{2,3}^{2}(n) \\
\quad+18 f_{2,3}^{4}(n) g_{2,3}(n) h_{2,3}(n)+142 f_{2,3}^{3}(n) g_{2,3}^{3}(n) \\
g_{2,3}(n+1)=\quad f_{2,3}^{5}(n) g_{2,3}(n)+f_{2,3}^{5}(n) h_{2,3}(n)+16 f_{2,3}^{4}(n) g_{2,3}^{2}(n)+18 f_{2,3}^{4}(n) g_{2,3}(n) h_{2,3}(n) \\
+89 f_{2,3}^{3}(n) g_{2,3}^{3}(n)+2 f_{2,3}^{4}(n) h_{2,3}^{2}(n)+77 f_{2,3}^{3}(n) g_{2,3}^{2}(n) h_{2,3}(n) \\
\quad+171 f_{2,3}^{2}(n) g_{2,3}^{4}(n), \\
\quad 3 f_{2,3}^{4}(n) g_{2,3}^{2}(n)+6 f_{2,3}^{4}(n) g_{2,3}(n) h_{2,3}(n)+51 f_{2,3}^{3}(n) g_{2,3}^{3}(n)+3 f_{2,3}^{4}(n) h_{2,3}^{2}(n) \\
+129 f_{2,3}^{3}(n) g_{2,3}^{2}(n) h_{2,3}(n)+279 f_{2,3}^{2}(n) g_{2,3}^{4}(n)+60 f_{2,3}^{3}(n) g_{2,3}(n) h_{2,3}^{2}(n) \\
+564 f_{2,3}^{2}(n) g_{2,3}^{3}(n) h_{2,3}(n)+468 f_{2,3}(n) g_{2,3}^{5}(n) .
\end{gathered}
$$

The figures for these configurations are too many to be shown here. Some values of $f_{2,3}(n), g_{2,3}(n)$, $h_{2,3}(n)$ are listed in Table 3 . These numbers grow exponentially, and do not have simple integer factorizations.

Tab. 3: The first few values of $f_{2,3}(n), g_{2,3}(n), h_{2,3}(n)$.

\begin{tabular}{|c||r|r|r|}
\hline \hline$n$ & 0 & 1 & 2 \\
\hline \hline$f_{2,3}(n)$ & 4 & 56,192 & $1,292,237,078,102,059,106,775,347,494,912$ \\
\hline$g_{2,3}(n)$ & 1 & 24,624 & $1,015,755,670,321,368,497,188,308,516,864$ \\
\hline$h_{2,3}(n)$ & 1 & 33,792 & $2,465,934,182,960,517,405,173,530,755,072$ \\
\hline \hline
\end{tabular}

The sequences of the ratios $\left\{\alpha_{2,3}(n)\right\}_{n=1}^{\infty}$ and $\left\{\beta_{2,3}(n)\right\}_{n=1}^{\infty}$ defined in Eq. (8) again decrease monotonically with $\lim _{n \rightarrow \infty} \alpha_{2,3}(n)=\beta_{2,3}(n)=0$. The ratio $\alpha_{2,3}(n) / \beta_{2,3}(n)$ decreases from four to three, the same as the results for $S G_{2}(n)$. The values of $\alpha_{2,3}(n), \beta_{2,3}(n)$ for small $n$ are listed in Table 4

By the same method as in Lemma 3.5, we have the general expression for the number of connected spanning subgraphs.

$$
f_{2,3}(n)=f_{2,3}(m)^{\frac{3}{5}\left(4 \times 6^{n-m-1}+1\right)} g_{2,3}(m)^{\frac{3}{5}\left(6^{n-m}-1\right)} \prod_{i=1}^{n-m} P_{2,3}(n-j)^{\frac{3}{5}\left(4 \times 6^{i-2}+1\right)}
$$


Tab. 4: The first few values of $\alpha_{2,3}(n), \beta_{2,3}(n)$. The last digits given are rounded off.

\begin{tabular}{|c||r|r|r|r|r|}
\hline \hline$n$ & 0 & 1 & 2 & 3 & 4 \\
\hline \hline$\alpha_{2,3}(n)$ & 4 & 2.28200129954516 & 1.27219282732945 & 0.660858801678112 & 0.326587785819904 \\
\hline$\beta_{2,3}(n)$ & 1 & 0.728693181818182 & 0.411915158701392 & 0.215917449918629 & 0.107573237878269 \\
\hline \hline
\end{tabular}

$$
\times \prod_{j=2}^{n-m} Q_{2,3}(n-j)^{\frac{3}{5}\left(6^{j-1}-1\right)}
$$

where

$$
\begin{gathered}
P_{2,3}(m)=142+18 \frac{\alpha_{2,3}(m)}{\beta_{2,3}(m)}+78 \alpha_{2,3}(m)+3 \frac{\alpha_{2,3}^{2}(m)}{\beta_{2,3}(m)}+15 \alpha_{2,3}^{2}(m)+\alpha_{2,3}^{3}(m) \\
Q_{2,3}(m)=171+77 \frac{\alpha_{2,3}(m)}{\beta_{2,3}(m)}+2 \frac{\alpha_{2,3}^{2}(m)}{\beta_{2,3}^{2}(m)}+89 \alpha_{2,3}(m)+18 \frac{\alpha_{2,3}^{2}(m)}{\beta_{2,3}(m)}+16 \alpha_{2,3}^{2}(m)+\frac{\alpha_{2,3}^{3}(m)}{\beta_{2,3}(m)}+\alpha_{2,3}^{3}(m)
\end{gathered}
$$

By the same argument given in Lemma 3.6 we have the upper and lower bounds of the asymptotic growth constant for the number of connected spanning subgraphs on $S G_{2,3}(n)$ :

$$
\begin{aligned}
& \quad \frac{1}{7 \times 6^{m}}\left[2 \ln f_{2,3}(m)+3 \ln g_{2,3}(m)\right]+\frac{1}{35 \times 6^{m}}[2 \ln 196+3 \ln 420] \leq z_{S G_{2,3}} \\
& \leq \frac{1}{7 \times 6^{m}}\left[2 \ln f_{2,3}(m)+3 \ln g_{2,3}(m)\right]+\frac{1}{35 \times 6^{m}}\left[2 \ln P_{2,3}(m)+3 \ln Q_{2,3}(m)\right],
\end{aligned}
$$

with $m$ a positive integer. We have the following proposition.

Proposition 4.1 The asymptotic growth constant for the number of connected spanning subgraphs on the two-dimensional Sierpinski gasket $S G_{2,3}(n)$ in the large n limit exists with value $z_{S G_{2,3}}=1.3972789680 \ldots$.

Similar to the argument given at the end of Section 3 , we find that both $(15 / 7)^{n} \alpha_{2,3}(n)$ and $(15 / 7)^{n} \beta_{2,3}(n)$ tend to (positive, finite) limits, such that

$$
\begin{aligned}
f_{2,3}(n) & \sim C\left(f_{2,3}\right)\left(\frac{7}{15}\right)^{(3 / 5) n} A_{2,3}^{6^{n}}, \quad g_{2,3}(n) \sim C\left(g_{2,3}\right)\left(\frac{15}{7}\right)^{(2 / 5) n} A_{2,3}^{6^{n}}, \\
h_{2,3}(n) & \sim C\left(h_{2,3}\right)\left(\frac{15}{7}\right)^{(7 / 5) n} A_{2,3}^{6^{n}},
\end{aligned}
$$

where $C\left(f_{2,3}\right), C\left(g_{2,3}\right), C\left(h_{2,3}\right)$ are constants with $z_{S G_{2,3}}=(5 / 7) \ln A_{2,3}$.

For $S G_{2,4}(n)$, the numbers of edges and vertices are given by

$$
e\left(S G_{2,4}(n)\right)=3 \times 10^{n},
$$




$$
v\left(S G_{2,4}(n)\right)=\frac{4 \times 10^{n}+5}{3},
$$

where again the three outmost vertices have degree two. There are $\left(10^{n}-1\right) / 3$ vertices of $S G_{2,4}(n)$ with degree six, and $\left(10^{n}-1\right)$ vertices with degree four. The initial values for the number of connected spanning subgraphs are the same as for $S G_{2}: f_{2,4}(0)=4, g_{2,4}(0)=1$ and $h_{2,4}(0)=1$. We wrote a computer program to obtain the recursion relations for $S G_{2,4}(n)$. They are lengthy and given in the appendix. Some values of $f_{2,4}(n), g_{2,4}(n), h_{2,4}(n)$ are listed in Table 5 . These numbers grow exponentially, and do not have simple integer factorizations.

Tab. 5: The first few values of $f_{2,4}(n), g_{2,4}(n), h_{2,4}(n)$.

\begin{tabular}{|c||r|r|}
\hline \hline$n$ & 1 & 2 \\
\hline \hline$f_{2,4}(n)$ & $164,119,040$ & $27,140,375,625,882,898,681,725,275,604,427,985,839,201,951,967,246,962,831,668,354,270,630,763,784,348,631,040,000$ \\
\hline$g_{2,4}(n)$ & $77,622,016$ & $25,675,411,803,142,714,297,950,351,525,972,498,833,548,895,007,181,465,816,231,861,389,426,797,930,493,247,488,000$ \\
\hline$h_{2,4}(n)$ & $112,848,672$ & $74,273,341,808,825,211,957,637,724,253,224,196,638,029,720,486,058,269,503,940,976,372,670,504,798,196,334,592,000$ \\
\hline \hline
\end{tabular}

The sequences of the ratios $\left\{\alpha_{2,4}(n)\right\}_{n=1}^{\infty}$ and $\left\{\beta_{2,4}(n)\right\}_{n=1}^{\infty}$ defined in Eq. 8, again decrease monotonically with $\lim _{n \rightarrow \infty} \alpha_{2,4}(n)=\beta_{2,4}(n)=0$. The ratio $\alpha_{2,4}(n) / \beta_{2,4}(n)$ decreases from four to three. The values of $\alpha_{2,4}(n), \beta_{2,4}(n)$ for small $n$ are listed in Table 6

Tab. 6: The first few values of $\alpha_{2,4}(n), \beta_{2,4}(n)$. The last digits given are rounded off.

\begin{tabular}{|c||r|r|r|r|r|}
\hline \hline$n$ & 0 & 1 & 2 & 3 & 4 \\
\hline \hline$\alpha_{2,4}(n)$ & 4 & 2.11433622131123 & 1.05705707211134 & 0.475214294459902 & 0.199993476915309 \\
\hline$\beta_{2,4}(n)$ & 1 & 0.687841643364664 & 0.345688118749653 & 0.156412715166630 & 0.662275943767262 \\
\hline \hline
\end{tabular}

By the same method as in Lemma 3.5, we have the general expression for the number of connected spanning subgraphs.

$$
f_{2,4}(n)=f_{2,4}(m)^{\frac{1}{3}\left(10^{n-m}+2\right)} g_{2,4}(m)^{\frac{2}{3}\left(10^{n-m}-1\right)} \prod_{i=1}^{n-m} P_{2,4}(n-j)^{\frac{1}{3}\left(10^{i-1}+2\right)} \prod_{j=2}^{n-m} Q_{2,4}(n-j)^{\frac{2}{3}\left(10^{j-1}-1\right)}
$$

where

$$
P_{2,4}(m)=11354+5856 \frac{\alpha_{2,4}(m)}{\beta_{2,4}(m)}+516 \frac{\alpha_{2,4}^{2}(m)}{\beta_{2,4}^{2}(m)}+2 \frac{\alpha_{2,4}^{3}(m)}{\beta_{2,4}^{3}(m)}+13626 \alpha_{2,4}(m)+4140 \frac{\alpha_{2,4}^{2}(m)}{\beta_{2,4}(m)}
$$




$$
\begin{aligned}
+ & 174 \frac{\alpha_{2,4}^{3}(m)}{\beta_{2,4}^{2}(m)}+6936 \alpha_{2,4}^{2}(m)+1140 \frac{\alpha_{2,4}^{3}(m)}{\beta_{2,4}(m)}+15 \frac{\alpha_{2,4}^{4}(m)}{\beta_{2,4}^{2}(m)}+1928 \alpha_{2,4}^{3}(m) \\
+ & 144 \frac{\alpha_{2,4}^{4}(m)}{\beta_{2,4}(m)}+309 \alpha_{2,4}^{4}(m)+7 \frac{\alpha_{2,4}^{5}(m)}{\beta_{2,4}(m)}+27 \alpha_{2,4}^{5}(m)+\alpha_{2,4}^{6}(m) \\
Q_{2,4}(m)= & 13732+14480 \frac{\alpha_{2,4}(m)}{\beta_{2,4}(m)}+2786 \frac{\alpha_{2,4}^{2}(m)}{\beta_{2,4}^{2}(m)}+82 \frac{\alpha_{2,4}^{3}(m)}{\beta_{2,4}^{3}(m)}+16250 \alpha_{2,4}(m) \\
+ & 10609 \frac{\alpha_{2,4}^{2}(m)}{\beta_{2,4}(m)}+1095 \frac{\alpha_{2,4}^{3}(m)}{\beta_{2,4}^{2}(m)}+12 \frac{\alpha_{2,4}^{4}(m)}{\beta_{2,4}^{3}(m)}+8015 \alpha_{2,4}^{2}(m)+3130 \frac{\alpha_{2,4}^{3}(m)}{\beta_{2,4}(m)} \\
+ & 142 \frac{\alpha_{2,4}^{4}(m)}{\beta_{2,4}^{2}(m)}+2148 \alpha_{2,4}^{3}(m)+462 \frac{\alpha_{2,4}^{4}(m)}{\beta_{2,4}(m)}+6 \frac{\alpha_{2,4}^{5}(m)}{\beta_{2,4}^{2}(m)}+332 \alpha_{2,4}^{4}(m) \\
+ & 34 \frac{\alpha_{2,4}^{5}(m)}{\beta_{2,4}(m)}+28 \alpha_{2,4}^{5}(m)+\frac{\alpha_{2,4}^{6}(m)}{\beta_{2,4}(m)}+\alpha_{2,4}^{6}(m) .
\end{aligned}
$$

By the same argument given in Lemma 3.6 , we have the upper and lower bounds of the asymptotic growth constant for the number of connected spanning subgraphs on $S G_{2,4}(n)$ :

$$
\begin{aligned}
& \frac{1}{4 \times 10^{m}}\left[\ln f_{2,4}(m)+2 \ln g_{2,4}(m)\right]+\frac{1}{36 \times 10^{m}}[\ln 33620+2 \ln 84460] \leq z_{S G_{2,4}} \\
& \leq \frac{1}{4 \times 10^{m}}\left[\ln f_{2,4}(m)+2 \ln g_{2,4}(m)\right]+\frac{1}{36 \times 10^{m}}\left[\ln P_{2,4}(m)+2 \ln Q_{2,4}(m)\right]
\end{aligned}
$$

with $m$ a positive integer. We have the following proposition.

Proposition 4.2 The asymptotic growth constant for the number of connected spanning subgraphs on the two-dimensional Sierpinski gasket $S G_{2,4}(n)$ in the large n limit exists with value $z_{S G_{2,4}}=1.484911260 \ldots$.

Similar to the argument given at the end of Section 3 , we find that both $(103 / 41)^{n} \alpha_{2,4}(n)$ and $(103 / 41)^{n} \beta_{2,4}(n)$ tend to (positive, finite) limits, such that

$$
\begin{aligned}
f_{2,4}(n) & \sim C\left(f_{2,4}\right)\left(\frac{41}{103}\right)^{(2 / 3) n} A_{2,4}^{10^{n}}, \quad g_{2,4}(n) \sim C\left(g_{2,4}\right)\left(\frac{103}{41}\right)^{n / 3} A_{2,4}^{10^{n}}, \\
h_{2,4}(n) & \sim C\left(h_{2,4}\right)\left(\frac{103}{41}\right)^{(4 / 3) n} A_{2,4}^{10^{n}},
\end{aligned}
$$

where $C\left(f_{2,4}\right), C\left(g_{2,4}\right), C\left(h_{2,4}\right)$ are constants with $z_{S G_{2,4}}=(3 / 4) \ln A_{2,4}$.

\section{The number of connected spanning subgraphs on $S G_{d}(n)$ with $d=3,4$}

In this section, we derive the asymptotic growth constant of connected spanning subgraphs on $S G_{d}(n)$ with $d=3,4$. For the three-dimensional Sierpinski gasket $S G_{3}(n)$, we use the following definitions. 
Definition 5.1 Consider the three-dimensional Sierpinski gasket $S G_{3}(n)$ at stage $n$.

(i) Define $f_{3}(n) \equiv N_{C S S G}\left(S G_{3}(n)\right)$ as the number of connected spanning subgraphs.

(ii) Define $g_{3}(n)$ as the number of spanning subgraphs with two components such that one certain outmost vertices belongs to one component and the other three outmost vertices belong to another component.

(iii) Define $h_{3}(n)$ as the number of spanning subgraphs with two components such that two certain outmost vertices belong to one component and the other two outmost vertices belong to another component.

(iv) Define $r_{3}(n)$ as the number of spanning subgraphs with three components such that two certain outmost vertices belong to one component and the other two outmost vertices separately belong to other components.

(v) Define $s_{3}(n)$ as the number of spanning subgraphs with four components such that each of the outmost vertices belongs to a different component.

The quantities $f_{3}(n), g_{3}(n), h_{3}(n), r_{3}(n)$ and $s_{3}(n)$ are illustrated in Fig. 7, where only the outmost vertices are shown. There are four different classes of connected subgraphs enumerated by $g_{3}(n)$, three classes enumerated by $h_{3}(n)$, and six classes enumerated by $r_{3}(n)$. The initial values at stage zero are $f_{3}(0)=38, g_{3}(0)=4, h_{3}(0)=1, r_{3}(0)=1$ and $s_{3}(0)=1$.
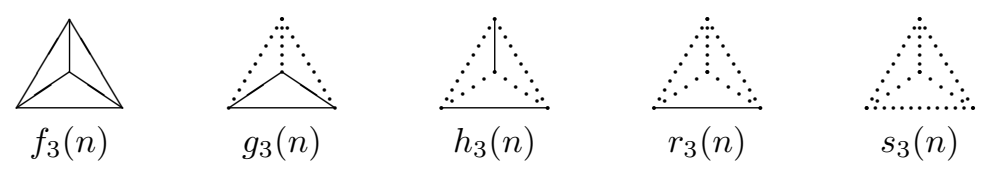

Fig. 7: Illustration for the spanning subgraphs $f_{3}(n), g_{3}(n), h_{3}(n), r_{3}(n)$ and $s_{3}(n)$. The two outmost vertices at the ends of a solid line belong to one component, while the two outmost vertices at the ends of a dot line belong to separated components.

The recursion relations are lengthy and given in the appendix. Some values of $f_{3}(n), g_{3}(n), h_{3}(n)$, $r_{3}(n), s_{3}(n)$ are listed in Table 7. These numbers grow exponentially, and do not have simple integer factorizations.

We find it is difficult to derive the bounds of the asymptotic growth constant for the number of connected spanning subgraphs on $S G_{3}(n)$. We calculate $f_{3}(m)$ up to $m=10$, and fit the numerical value of the asymptotic growth constant to have the following proposition.

Proposition 5.1 The asymptotic growth constant for the number of connected spanning subgraphs on the three-dimensional Sierpinski gasket $S G_{3}(n)$ in the large $n$ limit exists with value $z_{S G_{3}}=2.0637052 \ldots$. 
Tab. 7: The first few values of $f_{3}(n), g_{3}(n), h_{3}(n), r_{3}(n), s_{3}(n)$.

\begin{tabular}{|c||r|r|r|}
\hline \hline$n$ & 0 & 1 & 2 \\
\hline \hline$f_{3}(n)$ & 38 & $8,554,560$ & $25,988,410,915,610,195,960,527,441,920$ \\
\hline$g_{3}(n)$ & 4 & $1,271,416$ & $4,544,490,996,892,396,578,747,598,336$ \\
\hline$h_{3}(n)$ & 1 & 39,502 & $73,629,059,909,730,939,289,401,696$ \\
\hline$r_{3}(n)$ & 1 & 254,462 & $917,115,147,969,863,922,701,973,216$ \\
\hline$s_{3}(n)$ & 1 & 153,824 & $637,427,406,318,067,141,227,862,784$ \\
\hline \hline
\end{tabular}

For the four-dimensional Sierpinski gasket $S G_{4}(n)$, we use the following definitions.

Definition 5.2 Consider the four-dimensional Sierpinski gasket $S G_{4}(n)$ at stage $n$.

(i) Define $f_{4}(n) \equiv N_{C S S G}\left(S G_{4}(n)\right)$ as the number of connected spanning subgraphs.

(ii) Define $g_{4}(n)$ as the number of spanning subgraphs with two components such that two certain outmost vertices belong to one component and the other three outmost vertices belong to another component.

(iii) Define $g_{4}^{\prime}(n)$ as the number of spanning subgraphs with two components such that one certain outmost vertices belong to one component and the other four outmost vertices belong to another component.

(iv) Define $h_{4}(n)$ as the number of spanning subgraphs with three components such that one certain outmost vertices belong to one component, two certain other outmost vertices belong to another component and the remaining two outmost vertices belong to a third component.

(v) Define $h_{4}^{\prime}(n)$ as the number of spanning subgraphs with three components such that three certain outmost vertices belong to one component and the other two outmost vertices separately belong to other components.

(vi) Define $r_{4}(n)$ as the number of spanning subgraphs with four components such that two certain outmost vertices belong to one component and the other three outmost vertices separately belong to other components.

(vii) Define $s_{4}(n)$ as the number of spanning subgraphs with five components such that each of the outmost vertices belongs to a different component.

The quantities $f_{4}(n), g_{4}(n), g_{4}^{\prime}(n), h_{4}(n), h_{4}^{\prime}(n), r_{4}(n)$ and $s_{4}(n)$ are illustrated in Fig. 8 , where only the outmost vertices are shown. There are ten different classes of connected subgraphs enumerated by $g_{4}(n)$, five classes enumerated by $g_{4}^{\prime}(n)$, fifteen classes enumerated by $h_{4}(n)$, ten classes enumerated by $h_{4}^{\prime}(n)$ and ten classes enumerated by $r_{4}(n)$. The initial values at stage zero are $f_{4}(0)=728, g_{4}(0)=4$, $g_{4}^{\prime}(0)=38, h_{4}(0)=1, h_{4}^{\prime}(0)=4, r_{4}(0)=1$ and $s_{4}(0)=1$. 

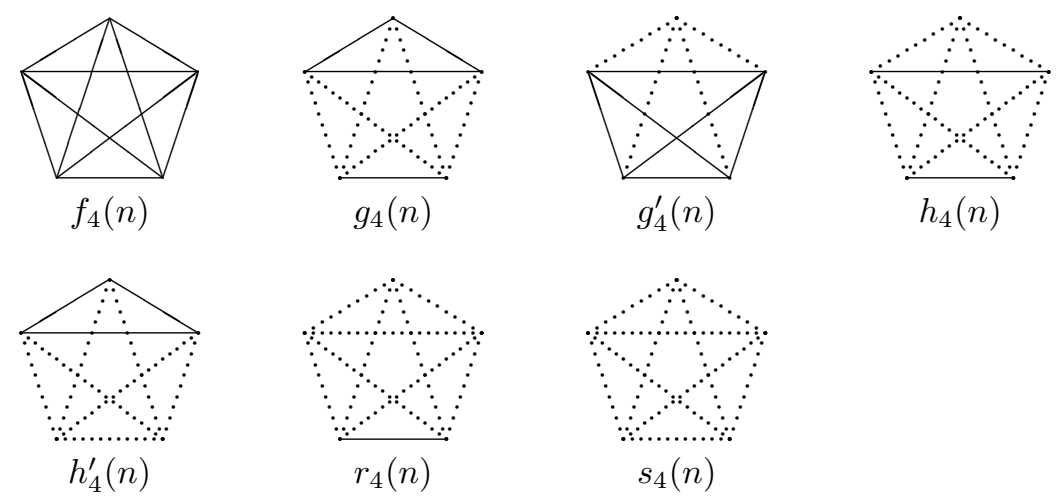

Fig. 8: Illustration for the spanning subgraphs $f_{4}(n), g_{4}(n), g_{4}^{\prime}(n), h_{4}(n), h_{4}^{\prime}(n), r_{4}(n)$ and $s_{4}(n)$. The two outmost vertices at the ends of a solid line belong to one component, while the two outmost vertices at the ends of a dot line belong to separated components.

We wrote a computer program to obtain the recursion relations for $S G_{4}(n)$. They are too lengthy to be included here, and are available from the authors on request. Some values of $f_{4}(n), g_{4}(n), g_{4}^{\prime}(n), h_{4}(n)$, $h_{4}^{\prime}(n), r_{4}(n), s_{4}(n)$ are listed in Table 8 . These numbers grow exponentially, and do not have simple integer factorizations.

Tab. 8: The first few values of $f_{4}(n), g_{4}(n), g_{4}^{\prime}(n), h_{4}(n), h_{4}^{\prime}(n), r_{4}(n), s_{4}(n)$.

\begin{tabular}{|c||r|r|}
\hline \hline$n$ & 1 & \\
\hline \hline$f_{4}(n)$ & $778,626,762,895,872$ & $1,024,406,418,765,003,907,906,096,145,114,250,200,136,082,865,744,856,739,402,552,777,712,697,606,144$ \\
\hline$g_{4}(n)$ & $88,489,486,528$ & $3,197,766,124,028,071,576,597,031,293,816,293,624,011,891,902,039,451,422,566,691,192,700,928$ \\
\hline$g_{4}^{\prime}(n)$ & $52,683,007,497,792$ & $69,863,645,008,967,428,965,504,302,095,638,435,727,081,373,061,446,549,243,263,724,682,869,735,424$ \\
\hline$h_{4}(n)$ & $15,626,116,736$ & $482,200,982,250,980,661,780,613,757,386,400,225,524,299,614,671,798,975,981,818,835,107,840$ \\
\hline$h_{4}^{\prime}(n)$ & $3,629,303,504,832$ & $4,765,691,494,696,738,414,627,738,223,389,422,884,987,040,021,355,690,863,179,839,765,575,892,992$ \\
\hline$r_{4}(n)$ & $258,767,297,696$ & $325,229,810,040,355,155,302,761,176,752,191,820,409,762,202,792,611,492,002,077,131,210,227,712$ \\
\hline$s_{4}(n)$ & $94,459,269,024$ & $110,974,534,976,153,854,286,043,758,382,592,092,762,465,813,295,695,669,459,295,951,908,765,696$ \\
\hline
\end{tabular}

It is even more difficult to derive the bounds of the asymptotic growth constant for the number of connected spanning subgraphs on $S G_{4}(n)$. We calculate $f_{4}(m)$ up to $m=6$, and we are satisfied with numerical fitting of the asymptotic growth constant to have the following proposition.

Proposition 5.2 The asymptotic growth constant for the number of connected spanning subgraphs on the four-dimensional Sierpinski gasket $S G_{4}(n)$ in the large $n$ limit exists with value $z_{S G_{4}}=2.7686025 \ldots$. 
Tab. 9: Numerical values of $\underline{z}_{S G_{d, b}}, \tilde{z}_{S G_{d, b}}, z_{S G_{d, b}}$, and their ratios. The last digits given are rounded off.

\begin{tabular}{|c|c|c|c|c|c|c|c|}
\hline \hline$d$ & $b$ & $D$ & $\underline{z}_{S G_{d, b}}$ & $\tilde{z}_{S G_{d, b}}$ & $z_{S G_{d, b}}$ & $\underline{z}_{S G_{d, b}} / z_{S G_{d, b}}$ & $\tilde{z}_{S G_{d, b}} / z_{S G_{d, b}}$ \\
\hline \hline 2 & 2 & 1.585 & 1.048594857 & 1.247337199 & 1.276495931 & 0.8214635326 & 0.9771572077 \\
\hline 2 & 3 & 1.631 & 1.133231895 & 1.312357559 & 1.397278968 & 0.8110276625 & 0.9392237265 \\
\hline 2 & 4 & 1.661 & 1.194401490 & 1.360516466 & 1.484911260 & 0.8043588344 & 0.9162274555 \\
\hline 3 & 2 & 2 & 1.569396409 & 1.666806281 & 2.063705 & 0.7604751 & 0.8076765 \\
\hline 4 & 2 & 2.322 & 1.914853265 & 1.981017076 & 2.768603 & 0.6916317 & 0.7155296 \\
\hline \hline
\end{tabular}

\section{Discussion}

Compared with the asymptotic growth constant for the number of spanning forests $N_{S F}$ on the Sierpinski gasket, defined as

$$
\tilde{z}_{G}=\lim _{v(G) \rightarrow \infty} \frac{\ln N_{S F}(G)}{v(G)}
$$

in Ref. [Chang and Chen(2008)], we find that $N_{C S S G}$ is larger than $N_{S F}$ for all the considered cases. We conjecture that this inequality holds for all the generalized Sierpinski gasket. We list the first few values of $\tilde{z}_{S G_{d, b}}, z_{S G_{d, b}}$, and their ratios in Table 9

As the spanning tree is a special case of connected spanning subgraphs where there is no cycles allowed, the number of spanning trees $N_{S T}(G)$ is always less than $N_{C S S G}(G)$. Define

$$
\underline{z}_{G}=\lim _{v(G) \rightarrow \infty} \frac{\ln N_{S T}(G)}{v(G)}
$$

then $\underline{z}_{G}<z_{G}$. We have obtained such asymptotic growth constants exactly for the number of spanning trees on the Sierpinski gasket $S G_{d}$ for general $d$ and $S G_{2, b}$ with $b=3,4$ in Ref. [Chang et al.(2007)]. They serve as the lower bounds for our current consideration for the connected spanning subgraphs. We list the first few values of $\underline{z}_{S G_{d, b}}, z_{S G_{d, b}}$, and their ratios in Table 9 . Notice that lower bound $\underline{z}_{S G_{d, b}}$ is closer to the exact value $z_{S G_{d, b}}$ when $d$ or $b$ is small, in contrast to the results for the spanning forests given in [Chang and Chen(2008)] that $\underline{z}_{S G_{d, b}}$ is relatively closer to $\tilde{z}_{S G_{d, b}}$ when $d$ or $b$ is large.

\section{Acknowledgements}

We would like to thank anonymous referees for valuable suggestions to improve the paper. The research of S.C.C. was partially supported by the NSC grant NSC-96-2112-M-006-001. The research of L.C.C was partially supported by TJ \& MY Foundation and NSC grant NSC 96-2115-M-030-002. L.C.C. would like to thank PIMS, university of British Columbia for the hospitality. 


\section{A Recursion relations for $S G_{2,4}(n)$}

We give the recursion relations for the generalized two-dimensional Sierpinski gasket $S G_{2,4}(n)$ here. Since the subscript is $(d, b)=(2,4)$ for all the quantities throughout this section, we will use the simplified notation $f_{n+1}$ to denote $f_{2,4}(n+1)$ and similar notations for other quantities. For any non-negative integer $n$, we have

$$
\begin{aligned}
f_{n+1}= & f_{n}^{10}+27 f_{n}^{9} g_{n}+7 f_{n}^{9} h_{n}+309 f_{n}^{8} g_{n}^{2}+144 f_{n}^{8} g_{n} h_{n}+1928 f_{n}^{7} g_{n}^{3}+15 f_{n}^{8} h_{n}^{2}+1140 f_{n}^{7} g_{n}^{2} h_{n} \\
& +6936 f_{n}^{6} g_{n}^{4}+174 f_{n}^{7} g_{n} h_{n}^{2}+4140 f_{n}^{6} g_{n}^{3} h_{n}+13626 f_{n}^{5} g_{n}^{5}+2 f_{n}^{7} h_{n}^{3}+516 f_{n}^{6} g_{n}^{2} h_{n}^{2} \\
& +5856 f_{n}^{5} g_{n}^{4} h_{n}+11354 f_{n}^{4} g_{n}^{6}, \\
g_{n+1}= & f_{n}^{9} g_{n}+f_{n}^{9} h_{n}+28 f_{n}^{8} g_{n}^{2}+34 f_{n}^{8} g_{n} h_{n}+332 f_{n}^{7} g_{n}^{3}+6 f_{n}^{8} h_{n}^{2}+462 f_{n}^{7} g_{n}^{2} h_{n}+2148 f_{n}^{6} g_{n}^{4} \\
& +142 f_{n}^{7} g_{n} h_{n}^{2}+3130 f_{n}^{6} g_{n}^{3} h_{n}+8015 f_{n}^{5} g_{n}^{5}+12 f_{n}^{7} h_{n}^{3}+1095 f_{n}^{6} g_{n}^{2} h_{n}^{2}+10609 f_{n}^{5} g_{n}^{4} h_{n} \\
& +16250 f_{n}^{4} g_{n}^{6}+82 f_{n}^{6} g_{n} h_{n}^{3}+2786 f_{n}^{5} g_{n}^{3} h_{n}^{2}+14480 f_{n}^{4} g_{n}^{5} h_{n}+13732 f_{n}^{3} g_{n}^{7}, \\
h_{n+1}=\quad & 3 f_{n}^{8} g_{n}^{2}+6 f_{n}^{8} g_{n} h_{n}+87 f_{n}^{7} g_{n}^{3}+3 f_{n}^{8} h_{n}^{2}+189 f_{n}^{7} g_{n}^{2} h_{n}+1068 f_{n}^{6} g_{n}^{4}+117 f_{n}^{7} g_{n} h_{n}^{2} \\
& +2558 f_{n}^{6} g_{n}^{3} h_{n}+7113 f_{n}^{5} g_{n}^{5}+15 f_{n}^{7} h_{n}^{3}+1869 f_{n}^{6} g_{n}^{2} h_{n}^{2}+17763 f_{n}^{5} g_{n}^{4} h_{n}+26934 f_{n}^{4} g_{n}^{6} \\
& +444 f_{n}^{6} g_{n} h_{n}^{3}+12756 f_{n}^{5} g_{n}^{3} h_{n}^{2}+61422 f_{n}^{4} g_{n}^{5} h_{n}+53826 f_{n}^{3} g_{n}^{7}+20 f_{n}^{6} h_{n}^{4}+2388 f_{n}^{5} g_{n}^{2} h_{n}^{3} \\
& +30948 f_{n}^{4} g_{n}^{4} h_{n}^{2}+83234 f_{n}^{3} g_{n}^{6} h_{n}+42210 f_{n}^{2} g_{n}^{8} .
\end{aligned}
$$

\section{B Recursion relations for $S G_{3}(n)$}

We give the recursion relations for the three-dimensional Sierpinski gasket $S G_{3}(n)$ here. Since the subscript is $d=3$ for all the quantities throughout this section, we will use the simplified notation $f_{n+1}$ to denote $f_{3}(n+1)$ and similar notations for other quantities. For any non-negative integer $n$, we have

$$
\begin{aligned}
f_{n+1}= & f_{n}^{4}+12 f_{n}^{3} g_{n}+12 f_{n}^{3} h_{n}+12 f_{n}^{3} r_{n}+48 f_{n}^{2} g_{n}^{2}+96 f_{n}^{2} g_{n} h_{n}+48 f_{n}^{2} h_{n}^{2}+72 f_{n}^{2} g_{n} r_{n} \\
& +72 f_{n}^{2} h_{n} r_{n}+56 f_{n} g_{n}^{3}+168 f_{n} g_{n}^{2} h_{n}+168 f_{n} g_{n} h_{n}^{2}+56 f_{n} h_{n}^{3}, \\
g_{n+1}= & f_{n}^{3} g_{n}+3 f_{n}^{3} r_{n}+9 f_{n}^{2} g_{n}^{2}+12 f_{n}^{2} g_{n} h_{n}+f_{n}^{3} s_{n}+36 f_{n}^{2} g_{n} r_{n}+30 f_{n}^{2} h_{n} r_{n}+28 f_{n} g_{n}^{3} \\
& +66 f_{n} g_{n}^{2} h_{n}+54 f_{n} g_{n} h_{n}^{2}+6 f_{n}^{2} g_{n} s_{n}+6 f_{n}^{2} h_{n} s_{n}+24 f_{n}^{2} r_{n}^{2}+108 f_{n} g_{n}^{2} r_{n} \\
& +192 f_{n} g_{n} h_{n} r_{n}+84 f_{n} h_{n}^{2} r_{n}+20 g_{n}^{4}+72 g_{n}^{3} h_{n}+96 g_{n}^{2} h_{n}^{2}+56 g_{n} h_{n}^{3}, \\
h_{n+1}= & 2 f_{n}^{2} h_{n}^{2}+4 f_{n}^{2} h_{n} r_{n}+12 f_{n} g_{n}^{2} h_{n}+12 f_{n} g_{n} h_{n}^{2}+16 f_{n} h_{n}^{3}+2 f_{n}^{2} r_{n}^{2}+12 f_{n} g_{n}^{2} r_{n}
\end{aligned}
$$




$$
\begin{array}{rl} 
& +48 f_{n} g_{n} h_{n} r_{n}+36 f_{n} h_{n}^{2} r_{n}+2 g_{n}^{4}+16 g_{n}^{3} h_{n}+36 g_{n}^{2} h_{n}^{2}+32 g_{n} h_{n}^{3}+22 h_{n}^{4}, \\
r_{n+1}= & f_{n}^{2} g_{n}^{2}+2 f_{n}^{2} h_{n}^{2}+6 f_{n}^{2} g_{n} r_{n}+6 f_{n}^{2} h_{n} r_{n}+6 f_{n} g_{n}^{3}+22 f_{n} g_{n}^{2} h_{n}+14 f_{n} g_{n} h_{n}^{2}+16 f_{n} h_{n}^{3} \\
+ & 2 f_{n}^{2} g_{n} s_{n}+2 f_{n}^{2} h_{n} s_{n}+12 f_{n}^{2} r_{n}^{2}+60 f_{n} g_{n}^{2} r_{n}+132 f_{n} g_{n} h_{n} r_{n}+66 f_{n} h_{n}^{2} r_{n}+12 g_{n}^{4} \\
+ & 52 g_{n}^{3} h_{n}+78 g_{n}^{2} h_{n}^{2}+48 g_{n} h_{n}^{3}+22 h_{n}^{4}+6 f_{n}^{2} r_{n} s_{n}+14 f_{n} g_{n}^{2} s_{n}+28 f_{n} g_{n} h_{n} s_{n} \\
+ & 14 f_{n} h_{n}^{2} s_{n}+120 f_{n} g_{n} r_{n}^{2}+120 f_{n} h_{n} r_{n}^{2}+88 g_{n}^{3} r_{n}+264 g_{n}^{2} h_{n} r_{n}+264 g_{n} h_{n}^{2} r_{n} \\
+ & 88 h_{n}^{3} r_{n}, \\
s_{n+1} & 4 f_{n} g_{n}^{3}+36 f_{n} g_{n}^{2} r_{n}+24 f_{n} g_{n} h_{n} r_{n}+12 g_{n}^{4}+24 g_{n}^{3} h_{n}+12 f_{n} g_{n}^{2} s_{n}+24 f_{n} g_{n} h_{n} s_{n} \\
& +12 f_{n} h_{n}^{2} s_{n}+144 f_{n} g_{n} r_{n}^{2}+120 f_{n} h_{n} r_{n}^{2}+144 g_{n}^{3} r_{n}+360 g_{n}^{2} h_{n} r_{n}+216 g_{n} h_{n}^{2} r_{n} \\
& +144 f_{n} g_{n} r_{n} s_{n}+144 f_{n} h_{n} r_{n} s_{n}+56 g_{n}^{3} s_{n}+168 g_{n}^{2} h_{n} s_{n}+168 g_{n} h_{n}^{2} s_{n}+56 h_{n}^{3} s_{n} \\
& +208 f_{n} r_{n}^{3}+720 g_{n}^{2} r_{n}^{2}+1440 g_{n} h_{n} r_{n}^{2}+720 h_{n}^{2} r_{n}^{2} .
\end{array}
$$




\section{References}

[Liu and Chow(1983)] C. I. Liu and Y. Chow, Enumeration of connected spanning subgraphs of a planar graph, Acta Math. Hung., 41: 27-36, 1983.

[Welsh(1993)] D. J. A. Welsh, Complexity: Knots, Colourings, and Counting (London Math. Soc. Lecture Notes series 186), Cambridge University Press, Cambridge, 1993.

[Shrock(2000)] R. Shrock, Exact Potts model partition functions on ladder graphs, Physica A, 283: 388446, 2000.

[Chang and Shrock(2000)] S.-C. Chang and R. Shrock, Exact Potts model partition function on strips of the triangular lattice, Physica A, 286: 189-238, 2000.

[Chang and Shrock(2001)(a)] S.-C. Chang and R. Shrock, Exact Potts model partition functions on strips of the honeycomb lattice, Physica A, 296: 183-233, 2001.

[Chang and Shrock(2001)(b)] S.-C. Chang and R. Shrock, Exact partition function for the Potts model with next-nearest neighbor couplings on arbitrary-length ladders, Int. J. Mod. Phys. B, 15: 443-478, 2001.

[Chang and Shrock(2001)(c)] S.-C. Chang and R. Shrock, Exact Potts model partition functions on wider arbitrary-length strips of the square lattice, Physica A, 296: 234-288, 2001.

[Mandelbrot(1982)] B. B. Mandelbrot, The Fractal Geometry of Nature, Freeman, San Francisco, 1982.

[Falconer(2003)] K. J. Falconer, Fractal Geometry: Mathematical Foundations and Applications, 2nd ed., Wiley, Chichester, 2003.

[Biggs(1993)] N. L. Biggs, Algebraic Graph Theory, 2nd ed., Cambridge University Press, Cambridge, 1993.

[Harary(1969)] F. Harary, Graph Theory, Addison-Wesley, New York, 1969.

[Gefen and Aharony(1981)] Y. Gefen and A. Aharony, Solvable fractal family, and its possible relation to the backbone at percolation, Phys. Rev. Lett., 47: 1771-1774, 1981.

[Hilfer and Blumen(1984)] R. Hilfer and A. Blumen, Renormalisation on Sierpinski-type fractals, $J$. Phys. A: Math. Gen., 17: L537-L545, 1984.

[Chang et al.(2007)] S.-C. Chang, L.-C. Chen and W.-S. Yang, Spanning trees on the Sierpinski gasket, J. Stat. Phys., 126: 649-667, 2007.

[Aho and Sloane(1973)] A. V. Aho and N. J. A. Sloane, Some doubly exponential sequences, Fibonacci Quart., 11: 429-437, 1973.

[Teufl and Wagner(2007)] E. Teufl and S. Wagner, Enumeration problems for classes of self-similar graphs, J. Combin. Theory Ser. A, 114: 1254-1277, 2007.

[Chang and Chen(2008)] S.-C. Chang and L.-C. Chen, Spanning forests on the Sierpinski gasket, discret. Math. Theor. Comput. Sci., 10: 55-76, 2008. 
